\title{
ON NORMAL GENERATION OF LINE BUNDLES ON ABELIAN THREEFOLDS
}

\author{
ELENA Rubei
}

\begin{abstract}
Let $X$ be a complex torus of dimension 3 and $L$ an ample line bundle of type $\left(2,2 d_{2}, 2 d_{3}\right)$ on $X$. In this paper we study when $L$ is normally generated.
\end{abstract}

\section{Introduction}

Let $X$ be a complex torus with an ample line bundle $L$ of type $\left(\delta_{1}, \ldots, \delta_{g}\right)$, with $\delta_{i} \mid \delta_{i+1}$, and let $\varphi_{L}$ be the associated rational map. In this paper we examine the problem whether $\varphi_{L}(X)$ is projectively normal in the case where $X$ is a threefold and $\delta_{1}=2$.

It is well known that if $\delta_{1} \geq 3$ and $X$ is of any dimension, then $\varphi_{L}$ is an embedding (Lefschetz' Theorem, see [Lef]) and $\varphi_{L}(X)$ is projectively normal (Koizumi's Theorem, see [Ko]). As to the case $\delta_{1}=1$, in [Laz], Lazarsfeld proved that, if $X$ is a complex torus of dimension 2, $L$ is an ample line bundle of type $(1, d)$ on $X,|L|$ has no fixed components and $\varphi_{L}$ is birational onto its image, then $\varphi_{L}(X)$ is projectively normal for $d$ odd $\geq 7$ and $d$ even $\geq 14$.

In the case of a complex torus with an ample line bundle $L$ of type $\left(2,2 d_{2}, \ldots, 2 d_{g}\right)$ we know that there exists an ample line bundle $M$, of type $\left(1, d_{2}, \ldots, d_{g}\right)$, such that $L=M^{2}$ (see for instance [L-B] Lemma 2.5.6) and in 1988 Ohbuchi proved the following theorem (see [Oh2]):

THEOREM (Ohbuchi). Let $M$ be an ample line bundle on a complex torus $X$. Suppose $c$ is a characteristic for $M$, then $M^{2}$ is normally generated if and only if no point of $t_{[c]}^{*} K\left(M^{2}\right)$ is a base point of $|M|$ (see below for the notations).

In $[R]$, using the quoted result of Ohbuchi, we proved the following theorem:

TheOREM (Rubei). Fix $d_{2}, \ldots, d_{g}$ positive integers with $d_{2}\left|d_{3}\right| \ldots \mid d_{g}$. Let $X$ be a complex torus of dimension $g$ and $M$ an ample line bundle on $X$ of type $\left(1, d_{2}, \ldots, d_{g}\right)$; set $L=M^{2}$. 
If $d_{i} \leq 2$ for every $i$, then $L$ is never normally generated (thus, if $\varphi_{L}$ is an embedding ${ }^{1}, \varphi_{L}(X)$ is not projectively normal). Otherwise (that is $d_{g}>2$ ) $L$ is normally generated for generic $\left(X, c_{1}(L)\right)$ in the moduli space of polarized abelian varieties of type $\left(2,2 d_{2}, \ldots, 2 d_{g}\right)$.

Here, using Ohbuchi's result, we determine, with the exception of some geometric situations, exactly which line bundles $L$ of type $\left(2,2 d_{2}, 2 d_{3}\right)$, with $d_{3}>2$, on a complex torus $X$ of dimension 3 are normally generated (Theorem 1), (see also Remark 1). We defer the statement of the theorem to $\$ 2$ and here we say only that, following the approach of Birkenhake-Lange-Ramanan's Theorem on global generation of primitive line bundles on abelian threefolds ([B-L-R]), we consider a line bundle $M$ of type $\left(1, d_{2}, d_{3}\right)$ with $L=M^{2}$ and an isogeny $\pi:(X, M) \rightarrow(Y, P)$ such that $\pi^{*} P=M$ and such that $\operatorname{ker} \pi$ is isomorphic to $Z / d_{2} \oplus Z / d_{3}$ and we divide the problem in the cases $(Y, P)$ is equal to the Jacobian of a Riemann surface of genus 3 , the product of the Jacobian of a Riemann surface of genus 2 and of an elliptic curve, or the product of three elliptic curves (see [L-B] $\S 10.8$ ).

To prove Theorem 1 we often use Birkenhake-Lange-Ramanan's Theorem on global generation of primitive line bundles on abelian threefolds. In proving Theorem 1, "incidentally" we prove also some small results about the global generation of primitive line bundles on abelian threefolds. We collect them in Proposition 1 glueing them with Lange-Birkenhake-Ramanan's Theorem.

One could conjecture that if $(X, M)$ is an abelian variety with a line bundle $M$ of type $\left(1, d_{2}, \ldots, d_{g}\right)$ with $d_{g}>2$ and $L=M^{2}$ is not normally generated then there exists an isogeny $(X, M) \rightarrow(Y, P)$ such that $\pi^{*} P=M$, ker $\pi$ is isomorphic to $\bigoplus_{i=2, \ldots, g} \boldsymbol{Z} / d_{i}$, and $(Y, P)$ is isomorphic to a nontrivial product of principally polarized abelian varieties. By a simple calculus of dimension we see easily that the conjecture is false (Remark 2).

Notation and Definitions. We collect here some notation and standard definitions we will use in all the paper.

- $\boldsymbol{X}, \boldsymbol{V}, \boldsymbol{\Lambda} . X$ is a complex torus equal to $V / \Lambda$ where $V$ is a complex vector space and $\Lambda$ a lattice in $V$.

- $X_{n} . X_{n}$ is the set of $n$-torsion points of $X$.

- $\varphi_{L} \cdot \varphi_{L}$ is the rational map associated to a line bundle $L$ on $X$.

- $\boldsymbol{t}_{\boldsymbol{x}}, t_{x}$ is the translation on $X$ by the point $x$.

- $\boldsymbol{A}_{t}$. If $A$ is a subset $X, A_{t}=A+t$.

- $\hat{X} . \hat{X}$ is the dual complex torus of $X$; it is isomorphic to $\operatorname{Pic}^{0}(X)$.

- $\phi_{L} . \phi_{L}$ is the homomorphism $X \rightarrow \hat{X}, x \mapsto t_{x}^{*} L \otimes L^{-1}$, where $L$ is a line bundle on $X$.

\footnotetext{
${ }^{1}$ We recall the following Ohbuchi's theorem (see [Oh1]): let $M$ be an ample line bundle on a complex torus $X ; M^{2}$ is very ample iff $(X, M)$ is not isomorphic to the product of two abelian varieties with line bundles $\left(X_{l}, M_{l}\right), l=1,2$, with $\operatorname{dim} X_{1}>0$ and $h^{0}\left(X_{1}, M_{1}\right)=1$.
} 
- $\boldsymbol{K}(\boldsymbol{L}), K(L)$ is the kernel of $\phi_{L}$; it does depend only on $H$, the first Chern class of $L$, thus we denote $K(L)$ also as $K(H)$; if $L$ is nondegenerate then $K(L)$ is a finite group isomorphic to $\left(\boldsymbol{Z} / d_{1} \oplus \cdots \oplus \boldsymbol{Z} / d_{g}\right)^{2}$ with $d_{i} \mid d_{i+1}$; we say that $L$ is of type $\left(d_{1}, \ldots, d_{g}\right)$. If $d_{1}=1$ we say that $L$ is primitive.

- $\boldsymbol{\Lambda}(\boldsymbol{L})$ or $\boldsymbol{\Lambda}(\boldsymbol{H}) \cdot \Lambda(L)=\Lambda(H)=\{v \in V \mid \operatorname{Im} H(v, \Lambda) \subset \boldsymbol{Z}\}$ where $L$ is a line bundle and $H$ its first Chern class; (we recall that $K(H)=\Lambda(H) / \Lambda$ ).

- $[\boldsymbol{y}] .[y]$ denotes the class in $X$ of a point $y \in V$.

- Suppose $H$ is a non degenerate hermitian form on $V, E=\operatorname{Im} H$ and $E(\Lambda, \Lambda) \subset \boldsymbol{Z}$.

A direct sum decomposition $\Lambda=\Lambda_{1} \oplus \Lambda_{2}$ is called a decomposition for $H$ (or for $E$ ) if $\Lambda_{1}$ and $\Lambda_{2}$ are isotropic with respect to $E$; a real vector space decomposition $V=V_{1} \oplus V_{2}$, with $V_{1}$ and $V_{2}$ real vector subspaces of $V$, is called a decomposition for $H$ (or for $E$ ) if $\left(V_{1} \cap \Lambda\right) \oplus\left(V_{2} \cap \Lambda\right)$ is a decomposition of $\Lambda$ for $H$.

Choose a decomposition of $V$ for $H: V=V_{1} \oplus V_{2}$. Let $L_{0}$ be the unique line bundle with Chern class $H$ and semicharacter $\chi_{0}: V \rightarrow C_{1}, \chi_{0}(v)=e^{\pi i E\left(v_{1}, v_{2}\right)}$, where $v=v_{1}+v_{2}$ and $v_{i} \in V_{l}$. For every $L$ with Chern class $H$ there is a point $c \in V$, uniquely determined up to translation by elements of $\Lambda(H)$, such that $L=t_{c c}^{*} L_{0}$ (see [L-B] Lemma 3.1.2); $c$ is called the characteristic of $L$ with respect to the chosen decomposition.

- A line bundle $L$ on $X$ is called symmetric if $(-1)_{X}^{*}(L) \simeq L$, where $(-1)_{X}$ is the multiplication by -1 on $X$. A line bundle $L$ with $c_{1}(L)=H$ is symmetric if and only if the characteristic of $L$ with respect to some decomposition of $V$ for $H$ is in $(1 / 2) \Lambda(H)$ (see [L-B], Chapter $4, \S 6$ and $\$ 7$, for a reference on symmetric bundles).

- A line bundle $L$ on $X$ is called normally generated if it is very ample and $\varphi_{L}(X)$ is projectively normal. We have that $L$ is normally generated iff it is ample and the natural maps $S^{n} H^{0}(X, L) \rightarrow H^{0}\left(X, L^{n}\right)$ are surjective for all $n \geq 2$ (see [L-B], Chapter $7, \S 3$ and $[\mathrm{M}]$, p. 38).

- $K_{C}, \boldsymbol{J}(\boldsymbol{C}), \boldsymbol{\mu}, V^{d}$. Let $C$ be a Riemann surface; $K_{C}$ denotes the canonical bundle on $C, J(C)$ denotes the Jacobian of $C, \mu: C^{(d)} \rightarrow J(C)$ denotes Abel's map and $V^{d}:=\mu\left(C^{(d)}\right)$.

- globally generated $=$ base point free

- p.p.a.v. = principally polarized abelian variety

\section{The main result}

We quote the following facts, lemmas and theorem from [B-L-R]; they will be useful to prove our result.

A polarized abelian variety $(X, M)$ of type $\left(d_{1}, \ldots, d_{g}\right)$ admits an isogeny onto a p.p.a.v. $\pi:(X, M) \rightarrow(Y, P)$ such that $\pi^{*} P=M$ and such that $\operatorname{ker} \pi$ (and then $\operatorname{ker} \hat{\pi}$, where $\hat{\pi}: \hat{Y} \rightarrow \hat{X}$ is the dual isogeny) is isomorphic to $\bigoplus_{i=1}^{g} \boldsymbol{Z} / d_{i}$. The isogeny $\pi$ determines the subgroup $Z:=\phi_{P}^{-1}(\operatorname{ker} \hat{\pi}) \simeq \bigoplus_{i=1}^{g} \boldsymbol{Z} / d_{i}$ in $Y$. Conversely any subgroup $Z$ of a p.p.a.v. $(Y, P)$ determines an isogeny $\pi: X \rightarrow Y:$ the dual of the isogeny $Y \simeq \hat{Y} \rightarrow \hat{X}:=Y / Z$. 
LEMMA 1 (Lemma 1.1 in [B-L-R]). Let $Z$ be a cyclic subgroup of order $d$ of a p.p.a.v. $(Y, P)$ and $\pi: X \rightarrow Y$ the associated isogeny. Then $M=\pi^{*}(P)$ is of type $(1, \ldots, 1, d)$.

LemMa 2 (part a) of Lemma (1.2) in [B-L-R]). Let $\pi:(X, M) \rightarrow(Y, P)$ be an isogeny onto a p.p.a.v. $(Y, P)$ associated to a finite subgroup $Z \subset Y$.

There is a canonical decomposition

$$
H^{0}(M) \simeq \bigoplus_{z \in Z} H^{0}\left(t_{z}^{*} P\right)
$$

induced by the embeddings $\pi^{*}: H^{0}\left(t_{z}^{*} P\right) \rightarrow H^{0}(M)$.

Besides we observe that $K(M)=\pi^{-1}(Z)$.

Lange-Birkenhake-Ramanan's Theorem on global generation of primitive line bundles on abelian threefolds (Theorem 1 in [B-L-R]). Let $X$ be a complex torus of dimension three and $M$ an ample line bundle on $X$ of type $(1,1, d)$ defining an irreducible polarization. Let $\pi:(X, M) \rightarrow(Y, P)$ be an isogeny with $\pi^{*} P=M,(Y, P)$ p.p.a.v. and such that $\operatorname{ker} \pi$ is isomorphic to $Z / d$. Let $Z:=$ $\phi_{P}^{-1}(\operatorname{ker} \hat{\pi}) \simeq Z / d=\langle z\rangle$ in $Y(\hat{\pi}: \hat{Y} \rightarrow \hat{X}$ the dual isogeny $)$.

a) Suppose $Y=J(C)$ is the Jacobian of a smooth curve $C$ of genus 3. If $d \geq 5$ and $C$ is not hyperelliptic, then $M$ is globally generated.

b) Suppose $Y=J(C) \times E$ is the product of the Jacobian of a smooth curve $C$ of genus 2 and of an elliptic curve $E$. Let $z=\left(z_{1}, z_{2}\right) \in J(C) \times E$. If $z_{1}$ is of order $\geq 5$, then $M$ is globally generated.

c) Suppose $Y=E_{1} \times E_{2} \times E_{3}$ is the product of three elliptic curves. Let $z=\left(z_{1}, z_{2}, z_{3}\right) \in E_{1} \times E_{2} \times E_{3}$. If $z_{l}$ is of order $\geq 4$ for $i=1,2,3$, then $M$ is globally generated.

We finish the preliminaries stating the following lemma:

LEMMA 3. Let $C$ be a Riemann surface of genus 2 and $z$ a point of order $d$ in $J(C)$ with $d \geq 3$. Then
a) $V^{1} \cap V_{z}^{1} \cap V_{2 z}^{1} \cap \cdots \cap V_{(d-1) z}^{1}=\emptyset$
b) $V^{1} \cap V_{z}^{1} \cap V_{2 z}^{1} \cap V_{3 z}^{1}=\emptyset$.

Proof. By a well-known theorem, $V^{1} \cap V_{s}^{1}$ is a set of two points counted with multiplicity if $s \neq 0$.

a) $V^{1} \cap V_{z}^{1} \cap V_{2 z}^{1} \cap \cdots \cap V_{(d-1) z}^{1}$ is invariant by $z$ thus if it is not empty then it contains at least $d$ points and this is absurd in fact $d \geq 3$.

b) Suppose $V^{1} \cap V_{z}^{1}=\{x, y\}$; then $V_{z}^{1} \cap V_{2 z}^{1}=\{x+z, y+z\}$ and $V_{2 z}^{1} \cap V_{3 z}^{1}=$ $\{x+2 z, y+2 z\}$. Thus we have $y=x+z$ and $y=x+2 z$ and then $z=0$, which is absurd. Observe that actually b) implies a).

Q.E.D. 
THEOREM 1. Let $X$ be a complex torus of dimension three and $M$ an ample line bundle on $X$ of type $\left(1, d_{2}, d_{3}\right)$ such that $d_{3}>2$; set $L=M^{2}$.

Let $\pi:(X, M) \rightarrow(Y, P)$ be an isogeny with $\pi^{*} P=M,(Y, P)$ p.p.a.v. and such that $\operatorname{ker} \pi$ (and then ker $\hat{\pi}$, where $\hat{\pi}: \hat{Y} \rightarrow \hat{X}$ is the dual isogeny) is isomorphic to $Z / d_{2} \oplus Z / d_{3}$. Let $Z:=\phi_{P}^{-1}(\operatorname{ker} \hat{\pi}) \simeq Z / d_{2} \oplus Z / d_{3}$ in $Y$.

1. Suppose first that $d_{2}=1$. Let $Z=\langle z\rangle$.

1a. Let $(Y, P)=\left(E_{1}, P_{1}\right) \times\left(E_{2}, P_{2}\right) \times\left(E_{3}, P_{3}\right)$, $E_{l}$ elliptic curves, $z=\left(z_{1}, z_{2}, z_{3}\right) \in$ $E_{1} \times E_{2} \times E_{3}$. Then $L$ is not normally generated if and only if we are in one of the following three cases:

- $\exists i$ such that $z_{l}=0$

- $\left\{\text { ord } z_{l}\right\}_{l=1,2,3}=\{2,4,4\}$

- $\left\{\text { ord } z_{l}\right\}_{l=1,2,3}=\{2,2, l\}$ for some $l \in N$.

1b. Let $(Y, P)=(J(C), \Theta) \times\left(E, P^{\prime}\right), E$ elliptic curve, $C$ Riemann surface of genus $2, z=\left(z_{1}, z_{2}\right) \in J(C) \times E$. Then $L$ is not normally generated if and only if we are in one of the following four cases:

- $\exists i$ such that $z_{\imath}=0$

- $\operatorname{ord}\left(z_{1}\right)=2$

- $\operatorname{ord}\left(z_{1}\right)=4$ and $\operatorname{ord}\left(z_{2}\right)=2$

- $\operatorname{ord}\left(z_{1}\right)=\operatorname{ord}\left(z_{2}\right)=3, C$ is a curve of the type $y^{2}=p(x)$ with $p(x)$ polynomial of degree 6 without multiple roots such that there exists a polynomial $q(x)$ of degree $\leq 3$ and $k \in C$ with $q(k) \neq 0$ such that $p(x)=q(x)^{2}+(x-k)^{6}$ and $z_{1}=$ $2 \mu(S)$ for a point $S$ with $x(S)=k$, where $\mu$ is the Abel map from a point of $C$ with $y=0 . \quad$ Observe that the set of the curves of the type we described above has codimension 1 in the moduli space of genus 2 curves.)

1c. Let $(Y, P)=(J(C), \Theta)$ with $C$ Riemann surface of genus 3 .

If $d_{3} \geq 5$ and $C$ is not hyperelliptic, $L$ is normally generated.

If $d_{3}=3$ and $C$ is not hyperelliptic, $L$ is not normally generated if and only if a) and b) hold:

a) $C$ is a smooth curve of the following type: choose a conic $\{q=0\}$ in $\boldsymbol{P}_{C}^{2}$ and four points on $\{q=0\}, T, Q, R, S$, with $\{T, Q\} \neq\{R, S\}$; let the line $T Q$ be $\left\{r_{1}=0\right\}$ and the line $R S$ be $\left\{r_{2}=0\right\}$; choose a polynomial $g$ of degree 2 such that, if $T \neq Q, g(T)=g(Q)=0$; let $C$ be the curve $\left\{r_{1} r_{2}^{3}+q g=0\right\}$.

b) $z=\mu(T+Q)-\mu(R+S)(T, Q, R, S$ in $a))$.

2. Suppose now that $\left.d_{2}\right\rangle 1$. Let $Z=\langle w\rangle \oplus\langle z\rangle$ with $\operatorname{ord}(w)=d_{2}$ and $\operatorname{ord}(z)=d_{3}$.

2a. Let $(Y, P)=\left(E_{1}, P_{1}\right) \times\left(E_{2}, P_{2}\right) \times\left(E_{3}, P_{3}\right), \quad E_{l}$ elliptic curves $z=\left(z_{1}, z_{2}, z_{3}\right) \epsilon$ $E_{1} \times E_{2} \times E_{3}, w=\left(w_{1}, w_{2}, w_{3}\right) \in E_{1} \times E_{2} \times E_{3}$.

- Suppose $z_{l} \neq 0 \forall i$. If $d_{3}>3$ then $L$ normally generated. If $d_{3}=3$ then $L$ is not normally generated if and only if $w=\left(\varepsilon_{1} z_{1}, \varepsilon_{2} z_{2}, \varepsilon_{3} z_{3}\right)$ with $\left\{\varepsilon_{1}, \varepsilon_{2}, \varepsilon_{3}\right\}=$ $\{0,1,-1\}$. 
- Suppose only one of the $z_{l}$ 's is equal to 0 , for instance $z_{3}=0$ and $z_{1} \neq 0$, $z_{2} \neq 0$. Then $L$ is not normally generated if and only if we are in one of the following five cases:
i) $w_{3}=0$;
ii) $\left\{\operatorname{ord}\left(z_{1}\right), \operatorname{ord}\left(z_{2}\right)\right\}=\{2,3\}, w_{1}=0$ and $w_{2}=0$;
iii) $\left\{\operatorname{ord}\left(z_{1}\right), \operatorname{ord}\left(z_{2}\right)\right\}=\{2,3\}$, ord $\left(w_{3}\right)=2$ and $\left\{\operatorname{ord}\left(w_{1}\right)\right.$, ord $\left.\left(w_{2}\right)\right\} \subseteq\{1,3\}$;
iv) $\left\{\operatorname{ord}\left(z_{1}\right), \operatorname{ord}\left(z_{2}\right)\right\}=\{2,3\}$, ord $\left(w_{3}\right)=3$ and $\left\{\operatorname{ord}\left(w_{1}\right)\right.$, ord $\left.\left(w_{2}\right)\right\} \subseteq\{1,2\}$;
v) $\left\{\operatorname{ord}\left(z_{1}\right), \operatorname{ord}\left(z_{2}\right)\right\}=\{2,3\},\left\{\operatorname{ord}\left(w_{1}\right), \operatorname{ord}\left(w_{2}\right), \operatorname{ord}\left(w_{3}\right)\right\} \subseteq\{1,2\}$.

- Suppose only two of the $z_{1}$ 's are equal to 0 , for instance $z_{2}=0$ and $z_{3}=0$ and $z_{1} \neq 0$. Then $L$ is not normally generated if and only if we are in one of the following four cases:
i) $w_{3}=0$;
ii) $w_{2}=0$;
iii) $\left\{\operatorname{ord}\left(w_{2}\right)\right.$, ord $\left.\left(w_{3}\right)\right\} \subseteq\{1,2\}$;
iv) $\left\{\operatorname{ord}\left(w_{2}\right), \operatorname{ord}\left(w_{3}\right)\right\} \subseteq\{2,3\}$.

2b. Let $(Y, P)=(J(C), \Theta) \times\left(E, P^{\prime}\right), E$ elliptic curve, $C$ Riemann surface of genus $2, z=\left(z_{1}, z_{2}\right) \in J(C) \times E, w=\left(w_{1}, w_{2}\right) \in J(C) \times E$. Then $L$ is not normally generated if and only if we are in one of the following two cases:

- $\operatorname{ord}\left(z_{1}\right)=2, \operatorname{ord}\left(z_{2}\right)>2$ and $w_{1}=0$

- $\operatorname{ord}\left(z_{1}\right)=4, \operatorname{ord}\left(z_{2}\right)=2, w_{2}=0$ and $w_{1}=2 z_{1}$.

2c. Let $(Y, P)=(J(C), \Theta)$ with $C$ not hyperelliptic Riemann surface of genus 3. If $d_{3} \geq 5$ then $L$ is normally generated.

Proof. Observe that, if $L^{\prime}$ is a line bundle with the same Chern class of $L$ (i.e. it is obtained from $L$ by a translation), then $L$ is normally generated if and only if $L^{\prime}$ is normally generated.

By one of Ohbuchi's theorems (see Introduction) $L=M^{2}$ is normally generated if and only if, once a decomposition of $V$ for $c_{1}(M)$ is fixed, $|M|$ has no base point in $t_{[c]}^{*} K\left(M^{2}\right)$ where $c \in V$ is a characteristic of $M$, i.e. there does not exist $b$ in the base locus of $|M|$ such that $2 b+2[c] \in K(M)$. In order to see when this condition holds we use the already quoted lemmas of the paper [B-L-R] (Lemma 1 and Lemma 2 in this paper).

1. Case $M$ is of type $\left(1,1, d_{3}\right)$

1a. Let $(Y, P)=\left(E_{1}, P_{1}\right) \times\left(E_{2}, P_{2}\right) \times\left(E_{3}, P_{3}\right), E_{l}$ elliptic curves, $z=\left(z_{1}, z_{2}, z_{3}\right) \in$ $E_{1} \times E_{2} \times E_{3}$. We claim that $L$ is normally generated if and only if $z_{l} \neq 0 \forall i$.

Let $\left(E_{l}, P_{l}\right)$ for $i=1,2,3$ be principally polarized elliptic curves with $P_{l}=$ (0); observe that the characteristic of $P$ is in $(1 / 2) \Lambda^{\prime}$, where $\Lambda^{\prime}$ is the lattice of $Y$, since $P$ is symmetric.

We recall that $K(M)=\pi^{-1}(\langle z\rangle)$ and the base locus of $|M|$ is the inverse image by $\pi$ of the base locus of $\oplus_{n} H^{0}\left(t_{n z}^{*} P\right)$.

Thus $L$ is normally generated if and only if $\exists b$ in the base locus of $\oplus_{n} H^{0}\left(t_{n z}^{*} P\right)$ such that $2 b \in\langle z\rangle$.

If $\exists i$ such that $z_{l}=0$, by Ohbuchi's theorem we recalled in the footnote 
of the Introduction, $L$ is not very ample and then it is not normally generated (in fact in this case obviously $\exists b$ in the base locus of $\bigoplus_{n} H^{0}\left(t_{n z}^{*} P\right)$ such that $2 b \in\langle z\rangle)$.

Let $z_{l} \neq 0$ for every $i$.

Let $d_{3}>3$; the base locus of $\bigoplus_{n} H^{0}\left(t_{n z}^{*} P\right)$ is the empty set if $\left\{\operatorname{ord} z_{l}\right\}_{l=1,2,3} \neq$ $\{2,4,4\}$ and $\neq\{2,2, l\}$ for any $l \in N$ (we can see this considering first the case ord $z_{l} \geq 3 \forall i$ and then the case $\exists i$ such that ord $z_{l}=2$ and then dividing the case $\exists i$ such that ord $z_{l}=2$ in the case $\exists j \neq i$ such that ord $z_{j}=2$ and the case $\nexists j \neq i$ such that ord $z_{J}=2$ ); if $\exists i, j$ with $i \neq j$ such that ord $z_{l}=\operatorname{ord} z_{j}$ $=2$, for instance ord $z_{1}=$ ord $z_{2}=2$, then the base locus of $\oplus_{n} H^{0}\left(t_{n z}^{*} P\right)$ is $\left(\{0\} \times\left\{z_{2}\right\} \times E_{3}\right) \cup\left(\left\{z_{1}\right\} \times\{0\} \times E_{3}\right)$, thus in this case $L$ is not normally generated, in fact $\exists b \in\left(\{0\} \times\left\{z_{2}\right\} \times E_{3}\right) \cup\left(\left\{z_{1}\right\} \times\{0\} \times E_{3}\right)$ such that $2 b \in\langle z\rangle$; if $\left\{\text { ord } z_{l}\right\}_{l=1,2,3}=\{2,4,4\}$ for instance $\left(\right.$ ord $z_{1}$, ord $z_{2}$, ord $\left.z_{3}\right)=(2,4,4)$, then the base locus of $\bigoplus_{n} H^{0}\left(t_{n z}^{*} P\right)$ is $\left(0, z_{2}, 3 z_{3}\right) \cup\left(0,3 z_{2}, z_{3}\right) \cup\left(z_{1}, 0,2 z_{3}\right) \cup\left(z_{1}, 2 z_{2}, 0\right)$ and again $L$ is not normally generated, because the double of everyone of these points is in $\langle z\rangle$.

Let $d_{3}=3$; then the base locus of $\bigoplus_{n} H^{0}\left(t_{n z}^{*} P\right)$ is the following set:

$$
\left\{\left(\sigma(1) z_{1}, \sigma(2) z_{2}, \sigma(3) z_{3}\right) \mid \sigma \in \mathscr{S}_{3}\right\} \text {. }
$$

Since $2\left(\sigma(1) z_{1}, \sigma(2) z_{2}, \sigma(3) z_{3}\right) \notin\langle z\rangle$ for all $\sigma \in \mathscr{S}_{3}$, we conclude that there is no base point of $|M|$ in $t_{[c]}^{*} K\left(M^{2}\right)$.

1b. Let $(Y, P)$ be the product of the Jacobian of a curve $C$ of genus 2 and of a principally polarized elliptic curve $\left(E, P^{\prime}\right)$ and let $z=\left(z_{1}, z_{2}\right) \in J(C) \times E$. If $\sigma$ is the hyperelliptic involution of $C$, choose one of the points that are fixed by $\sigma$ as the base point of Abel's map $\mu$. Sometimes it will be useful to see $C$ as the Riemann surface $y^{2}=p(x)$ with $p(x)$ polynomial of degree 6 without multiple roots; we will see $C$ as the Riemann surface obtained by glueing two copies of $\boldsymbol{P}^{1}$ as described for instance in [F-R] p. 145; we will denote the two points at infinity in the two copies of $\boldsymbol{P}^{1}$ by $P_{1}^{\infty}$ and $P_{2}^{\infty}$. We can suppose $(Y, P)=$ $\left(J(C), V^{1}\right) \times\left(E, P^{\prime}\right)$, with $P^{\prime}=(0)$.

We have to see whether there is a point $b$ in $\bigcap_{n}\left(\left(V^{1} \times E\right) \cup(J(C) \times\right.$ $\{0\}))_{n\left(z_{1}, z_{2}\right)}$ such that $2 b \in Z$ (in fact the characteristic of $P$ is in $(1 / 2) \Lambda^{\prime}$, where $\Lambda^{\prime}$ is the lattice of $Y$, since $P$ is symmetric).

Case ord $\left(z_{1}\right)=2$. Observe that

$$
\begin{aligned}
& \bigcap_{n}\left(\left(V^{1} \times E\right) \cup(J(C) \times\{0\})\right)_{n\left(z_{1}, z_{2}\right)} \\
& =\left(\left(V^{1} \times E\right) \cup(J(C) \times\{0\})\right) \cap\left(\left(V_{z_{1}}^{1} \times E\right) \cup\left(J(C) \times\left\{z_{2}\right\}\right)\right) \\
& \cap\left(\left(V^{1} \times E\right) \cup\left(J(C) \times\left\{2 z_{2}\right\}\right)\right) \cap\left(\left(V_{z_{1}}^{1} \times E\right) \cup\left(J(C) \times\left\{3 z_{2}\right\}\right)\right)
\end{aligned}
$$

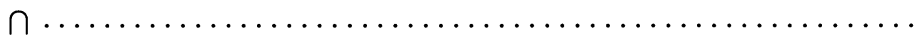

$$
\begin{aligned}
& =\left(V^{1} \times E\right) \cap\left(V_{z_{1}}^{1} \times E\right)=\left(V^{1} \cap V_{z_{1}}^{1}\right) \times E .
\end{aligned}
$$


We see that $\exists b \in\left(V^{1} \cap V_{z_{1}}^{1}\right) \times E$ such that $2 b \in Z$, in fact $\exists a \in V^{1} \cap V_{z_{1}}^{1}$ such that $2 a \in\left\langle z_{1}\right\rangle$; since $2 z_{1}=0$ one can write $z_{1}=\mu(R)-\mu(S)$ with $R$ and $S$ in the set of the points that are fixed by $\sigma$; thus we can take $a=\mu(R)$ because $\mu(R) \in V^{1} \cap V_{z_{1}}^{1}$ and $2 \mu(R)=0$.

Thus in this case $L$ is never normally generated.

Case ord $\left(z_{1}\right)=3$. We divide this case in two subcases depending on ord $\left(z_{2}\right)$. - $\operatorname{ord}\left(z_{2}\right)=2$ or $\operatorname{ord}\left(z_{2}\right)>3$.

Observe that

$$
\begin{aligned}
& \bigcap_{n}\left(\left(V^{1} \times E\right) \cup(J(C) \times\{0\})\right)_{n\left(z_{1}, z_{2}\right)} \\
& =\left(\left(V^{1} \times E\right) \cup(J(C) \times\{0\})\right) \cap\left(\left(V_{z_{1}}^{1} \times E\right) \cup\left(J(C) \times\left\{z_{2}\right\}\right)\right) \\
& \cap\left(\left(V_{2 z_{1}}^{1} \times E\right) \cup\left(J(C) \times\left\{2 z_{2}\right\}\right)\right) \\
& \cap\left(\left(V^{1} \times E\right) \cup\left(J(C) \times\left\{3 z_{2}\right\}\right)\right) \cap\left(\left(V_{z_{1}}^{1} \times E\right) \cup\left(J(C) \times\left\{4 z_{2}\right\}\right)\right) \\
& \cap\left(\left(V_{2 z_{1}}^{1} \times E\right) \cup\left(J(C) \times\left\{5 z_{2}\right\}\right)\right)
\end{aligned}
$$

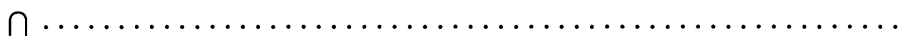

$$
\begin{aligned}
& =\left(V^{1} \times E\right) \cap\left(V_{z_{1}}^{1} \times E\right) \cap\left(V_{2 z_{1}}^{1} \times E\right)=\left(V^{1} \cap V_{z_{1}}^{1} \cap V_{2 z_{1}}^{1}\right) \times E .
\end{aligned}
$$

By Lemma 3, $V^{1} \cap V_{z_{1}}^{1} \cap V_{2 z_{1}}^{1}=\emptyset$. Thus $M$ is globally generated and $L$ is normally generated.

- $\operatorname{ord}\left(z_{2}\right)=3$.

Observe that

$$
\begin{aligned}
\bigcap_{n}(( & \left.\left.V^{1} \times E\right) \cup(J(C) \times\{0\})\right)_{n\left(z_{1}, z_{2}\right)} \\
= & \left(\left(V^{1} \times E\right) \cup(J(C) \times\{0\})\right) \cap\left(\left(V_{z_{1}}^{1} \times E\right) \cup\left(J(C) \times\left\{z_{2}\right\}\right)\right) \\
& \cap\left(\left(V_{2 z_{1}}^{1} \times E\right) \cup\left(J(C) \times\left\{2 z_{2}\right\}\right)\right) \\
= & \left(\left(V^{1} \cap V_{z_{1}}^{1} \cap V_{2 z_{1}}^{1}\right) \times E\right) \cup\left(\left(V^{1} \cap V_{z_{1}}^{1}\right) \times\left\{2 z_{2}\right\}\right) \\
& \cup\left(\left(V^{1} \cap V_{2 z_{1}}^{1}\right) \times\left\{z_{2}\right\}\right) \cup\left(\left(V_{z_{1}}^{1} \cap V_{2 z_{1}}^{1}\right) \times\{0\}\right) \\
= & \left(\left(V^{1} \cap V_{z_{1}}^{1}\right) \times\left\{2 z_{2}\right\}\right) \cup\left(\left(V^{1} \cap V_{2 z_{1}}^{1}\right) \times\left\{z_{2}\right\}\right) \cup\left(\left(V_{z_{1}}^{1} \cap V_{2 z_{1}}^{1}\right) \times\{0\}\right) \\
= & \left(\left(V^{1} \cap V_{z_{1}}^{1}\right) \times\left\{2 z_{2}\right\}\right) \cup\left(\left(V^{1} \cap V_{z_{1}}^{1}\right) \times\left\{2 z_{2}\right\}\right)_{2\left(z_{1}, z_{2}\right)} \cup\left(\left(V^{1} \cap V_{z_{1}}^{1}\right) \times\left\{2 z_{2}\right\}\right)_{\left(z_{1}, z_{2}\right)}
\end{aligned}
$$

where the last but one equality holds by Lemma 3 . 
Observe that: $\exists b \in V^{1} \cap V_{z_{1}}^{1} \times\left\{2 z_{2}\right\}$ such that $2 b \in Z \Leftrightarrow$ $\exists b \in\left(\left(V^{1} \cap V_{z_{1}}^{1}\right) \times\left\{2 z_{2}\right\}\right)_{2\left(z_{1}, z_{2}\right)}$ such that $2 b \in Z \Leftrightarrow$ $\exists b \in\left(\left(V^{1} \cap V_{z_{1}}^{1}\right) \times\left\{2 z_{2}\right\}\right)_{\left(z_{1}, z_{2}\right)}$ such that $2 b \in Z$.

Thus it is sufficient to check when $\exists b \in V^{1} \cap V_{z_{1}}^{1} \times\left\{2 z_{2}\right\}$ such that $2 b \in Z$. One can easily see that: $\exists b \in V^{1} \cap V_{z_{1}}^{1} \times\left\{2 z_{2}\right\}$ such that $2 b \in Z \Leftrightarrow$ $\exists a \in V^{1} \cap V_{z_{1}}^{1}$ such that $2 a=z_{1} \Leftrightarrow$ $\exists S, Q \in C$ such that $\mu(S)=\mu(Q)+z_{1}$ and $2 \mu(S)=z_{1} \Leftrightarrow$ $\exists S, Q \in C$ such that $\mu(S)+\mu(Q)=0$ and $2 \mu(S)=z_{1} \Leftrightarrow$ $\exists S, Q \in C$ such that $S+Q \in K_{C}$ and $2 \mu(S)=z_{1} \Leftrightarrow$ $\exists S \in C$ such that $2 \mu(S)=z_{1}(Q$ will be $\sigma(S))$.

Thus $L$ is not normally generated if and only if there is a point $S$ in $C$ such that it is not a ramification point and $6 \mu(S)=0$ (that is $6 S \in 3 K_{C}$ ) and $z_{1}=$ $2 \mu(S)$.

Observe that $3 P_{1}^{\infty}+3 P_{2}^{\infty} \in 3 K_{C}$ and $\mathscr{L}\left(3 P_{1}^{\infty}+3 P_{2}^{\infty}\right)=\left\langle 1, x, x^{2}, x^{3}, y\right\rangle=$ $\{\alpha y-q(x): \operatorname{deg} q \leq 3, \alpha \in \mathbf{C}\}$.

Thus there is a point $S$ in $C\left(=\left\{y^{2}=p(x)\right\}\right)$ such that it is not a ramification point and $6 S \in K_{C}$ if and only if $\exists q(x)$ of degree $\leq 3$ and $k \in C$ with $p(k) \neq 0$ such that $q(x)^{2}-p(x)$ is a scalar multiple of $(x-k)^{6}(S$ will be one of the two points with $x=k$ ). Observe that the set of the curves of this type has codimension 1 in the moduli space of genus 2 curves.

Thus we conclude the proof of this case. $\operatorname{ord}\left(z_{2}\right)$.

Case ord $\left(z_{1}\right)=4$. We divide this case in three subcases depending on - $\operatorname{ord}\left(z_{2}\right)=2$.

Observe that

$$
\begin{aligned}
\bigcap_{n}(( & \left.\left.V^{1} \times E\right) \cup(J(C) \times\{0\})\right)_{n\left(z_{1}, z_{2}\right)} \\
= & \left(\left(V^{1} \times E\right) \cup(J(C) \times\{0\})\right) \cap\left(\left(V_{z_{1}}^{1} \times E\right) \cup\left(J(C) \times\left\{z_{2}\right\}\right)\right) \\
& \cap\left(\left(V_{2 z_{1}}^{1} \times E\right) \cup(J(C) \times\{0\})\right) \cap\left(\left(V_{3 z_{1}}^{1} \times E\right) \cup\left(J(C) \times\left\{z_{2}\right\}\right)\right) \\
= & \left(\left(\left(V^{1} \cap V_{2 z_{1}}^{1}\right) \times E\right) \cup(J(C) \times\{0\})\right) \\
& \cap\left(\left(\left(V_{z_{1}}^{1} \cap V_{3 z_{1}}^{1}\right) \times E\right) \cup\left(J(C) \times\left\{z_{2}\right\}\right)\right) \\
= & \left(\left(V^{1} \cap V_{2 z_{1}}^{1}\right) \times\left\{z_{2}\right\}\right) \cup\left(\left(V_{z_{1}}^{1} \cap V_{3 z_{1}}^{1}\right) \times\{0\}\right) \\
& \cup\left(\left(V^{1} \cap V_{z_{1}}^{1} \cap V_{2 z_{1}}^{1} \cap V_{3 z_{1}}^{1}\right) \times E\right) \\
= & \left(\left(V^{1} \cap V_{2 z_{1}}^{1}\right) \times\left\{z_{2}\right\}\right) \cup\left(\left(V_{z_{1}}^{1} \cap V_{3 z_{1}}^{1}\right) \times\{0\}\right),
\end{aligned}
$$

where last equality holds by Lemma 3 . 
We see that $\exists b \in\left(V^{1} \cap V_{2 z_{1}}^{1}\right) \times\left\{z_{2}\right\}$ such that $2 b \in Z$, in fact $\exists a \in V^{1} \cap V_{2 z_{1}}^{1}$ such that $2 a=0$ : since $4 z_{1}=0$ one can write $2 z_{1}=\mu(R)+\mu(S)$ with $R$ and $S$ in the set of the points that are fixed by $\sigma$; thus we can take $a=\mu(R)$ because $\mu(R) \in V^{1} \cap V_{2 z_{1}}^{1}$ and $2 \mu(R)=0$.

Thus in this case $L$ is never normally generated.

- $\operatorname{ord}\left(z_{2}\right)=4$.

Observe that

$$
\begin{aligned}
\bigcap_{n}(( & \left.\left.V^{1} \times E\right) \cup(J(C) \times\{0\})\right)_{n\left(z_{1}, z_{2}\right)} \\
= & \left(\left(V^{1} \times E\right) \cup(J(C) \times\{0\})\right) \cap\left(\left(V_{z_{1}}^{1} \times E\right) \cup\left(J(C) \times\left\{z_{2}\right\}\right)\right) \\
& \cap\left(\left(V_{2 z_{1}}^{1} \times E\right) \cup\left(J(C) \times\left\{2 z_{2}\right\}\right)\right) \cap\left(\left(V_{3 z_{1}}^{1} \times E\right) \cup\left(J(C) \times\left\{3 z_{2}\right\}\right)\right) \\
= & \left(\left(V^{1} \cap V_{z_{1}}^{1} \cap V_{2 z_{1}}^{1} \cap V_{3 z_{1}}^{1}\right) \times E\right) \\
& \cup\left(\left(V^{1} \cap V_{z_{1}}^{1} \cap V_{2 z_{1}}^{1}\right) \times\left\{3 z_{2}\right\}\right) \cup\left(\left(V^{1} \cap V_{z_{1}}^{1} \cap V_{3 z_{1}}^{1}\right) \times\left\{2 z_{2}\right\}\right) \\
& \cup\left(\left(V^{1} \cap V_{2 z_{1}}^{1} \cap V_{3 z_{1}}^{1}\right) \times\left\{z_{2}\right\}\right) \cup\left(\left(V_{z_{1}}^{1} \cap V_{2 z_{1}}^{1} \cap V_{3 z_{1}}^{1}\right) \times\{0\}\right) \\
= & \left(\left(V^{1} \cap V_{z_{1}}^{1} \cap V_{2 z_{1}}^{1}\right) \times\left\{3 z_{2}\right\}\right) \cup\left(\left(V^{1} \cap V_{z_{1}}^{1} \cap V_{3 z_{1}}^{1}\right) \times\left\{2 z_{2}\right\}\right) \\
& \cup\left(\left(V^{1} \cap V_{2 z_{1}}^{1} \cap V_{3 z_{1}}^{1}\right) \times\left\{z_{2}\right\}\right) \cup\left(\left(V_{z_{1}}^{1} \cap V_{2 z_{1}}^{1} \cap V_{3 z_{1}}^{1}\right) \times\{0\}\right) \\
= & \bigcup_{n=0, \ldots 3}\left(\left(V^{1} \cap V_{z_{1}}^{1} \cap V_{2 z_{1}}^{1}\right) \times\left\{3 z_{2}\right\}\right)_{n\left(z_{1}, z_{2}\right)}
\end{aligned}
$$

where the last but one equality holds by Lemma 3 .

Obviously we can reduce to see whether $\exists a \in V^{1} \cap V_{z_{1}}^{1} \cap V_{2 z_{1}}^{1}$ such that $2\left(a, 3 z_{2}\right) \in Z$ that is $2 a=2 z_{1}$.

But it is not possible that $\exists S \in C$ such that $2 \mu(S)=2 z_{1}$, in fact, this implies that $2 \mu(S) \neq 0$, that is $S$ is not fixed by $\sigma$, and $4 \mu(S)=0$, that is $4 S \in 2 K_{C}$; but $2 K_{C} \ni 2 P_{1}^{\infty}+2 P_{2}^{\infty}, \mathscr{L}\left(2 P_{1}^{\infty}+2 P_{2}^{\infty}\right)=\left\langle 1, x, x^{2}\right\rangle$, thus $4 S \in 2 K_{C}$ implies $\exists q(x)$ of degree $\leq 2$ such that $(q)=4 S-2 P_{1}^{\infty}-2 P_{2}^{\infty}$ and this is absurd since $(q)$ and $2 P_{1}^{\infty}+2 P_{2}^{\infty}$ are symmetric for $\sigma$ while $4 S$ is not because $S$ is not fixed by $\sigma$.

Thus $L$ is normally generated.

- $\operatorname{ord}\left(z_{2}\right)=3$ or $\operatorname{ord}\left(z_{2}\right) \geq 5$.

Observe that

$$
\begin{aligned}
& \bigcap_{n}\left(\left(V^{1} \times E\right) \cup(J(C) \times\{0\})\right)_{n\left(z_{1}, z_{2}\right)} \\
&=\left(\left(V^{1} \times E\right) \cup(J(C) \times\{0\})\right) \cap\left(\left(V_{z_{1}}^{1} \times E\right) \cup\left(J(C) \times\left\{z_{2}\right\}\right)\right) \\
& \cap\left(\left(V_{2 z_{1}}^{1} \times E\right) \cup\left(J(C) \times\left\{2 z_{2}\right\}\right)\right) \cap\left(\left(V_{3 z_{1}}^{1} \times E\right) \cup\left(J(C) \times\left\{3 z_{2}\right\}\right)\right) \\
& \cap\left(\left(V^{1} \times E\right) \cup\left(J(C) \times\left\{4 z_{2}\right\}\right)\right) \cap\left(\left(V_{z_{1}}^{1} \times E\right) \cup\left(J(C) \times\left\{5 z_{2}\right\}\right)\right)
\end{aligned}
$$




$$
\begin{aligned}
& \cap\left(\left(V_{2 z_{1}}^{1} \times E\right) \cup\left(J(C) \times\left\{6 z_{2}\right\}\right)\right) \cap\left(\left(V_{3 z_{1}}^{1} \times E\right) \cup\left(J(C) \times\left\{7 z_{2}\right\}\right)\right) \\
& \cap \ldots \ldots \ldots \ldots \ldots \ldots \ldots \ldots \ldots \ldots \ldots \ldots \ldots \ldots \ldots \ldots \ldots \ldots \ldots \ldots \ldots \ldots \ldots \ldots \ldots \ldots \ldots \ldots \ldots \\
= & \left(V^{1} \times E\right) \cap\left(V_{z_{1}}^{1} \times E\right) \cap\left(V_{2 z_{1}}^{1} \times E\right) \cap\left(V_{3 z_{1}}^{1} \times E\right) \\
= & \left(V^{1} \cap V_{z_{1}}^{1} \cap V_{2 z_{1}}^{1} \cap V_{3 z_{1}}^{1}\right) \times E=\emptyset .
\end{aligned}
$$

where last equality holds by Lemma 3 .

Case ord $\left(z_{1}\right) \geq 5$. By Theorem 1 part b) in [B-L-R] (we recalled at the beginning of this section) $M$ is globally generated and then $L$ is normally generated.

1c. Let $(Y, P)=(J(C), \Theta)$ with $C$ Riemann surface of genus 3 .

If $C$ is not hyperelliptic and $d_{3} \geq 5$ then $|M|$ has not base points, by Theorem 1 in [B-L-R]. Thus $L$ is normally generated.

Suppose $C$ is not hyperelliptic and $d_{3}=3$. We want to see when $\exists b \in V^{2} \cap V_{z}^{2} \cap V_{2 z}^{2}$ such that $2 b \in Z+\mu\left(K_{C}\right)$. We can easily reduce to see when $\exists b \in V^{2} \cap V_{z}^{2} \cap V_{2 z}^{2}$ such that $2 b=\mu\left(K_{C}\right)$. This is equivalent to say $\exists T, Q, R, S, A, B \in C$ such that $\mu(T+Q)=\mu(R+S)+z=\mu(A+B)+2 z$ and $2 \mu(T+Q)=\mu\left(K_{C}\right)$.

This is equivalent to say $\exists T, Q, R, S, A, B \in C$ such that:

$R+S+A+B \in K_{C}, 2(T+Q) \in K_{C}, \mu(T+Q)-\mu(R+S)$ is of order 3.

This is equivalent to say $\exists T, Q, R, S, A, B \in C$ such that:

$R+S+A+B \in K_{C}, 2(T+Q) \in K_{C}, T+Q+A+B \sim 2(R+S)$.

This is equivalent to say $\exists T, Q, R, S, A, B \in C$ such that:

$R+S+A+B \in K_{C}, 2(T+Q) \in K_{C}, T+Q+3(R+S) \in 2 K_{C}$.

If $v: C \rightarrow H^{0}\left(C, K_{C}\right)^{*}$ is the canonical map, this is equivalent to say that $\exists T, Q, R, S, A, B \in C$ such that $v(R), v(S), v(A), v(B)$ are in the same line, the line $v(T) v(Q)$ is bitangent to $v(C)$ in $v(T)$ and $v(Q)$ there is a conic that cuts on $v(C)$ the divisor $T+Q+3(R+S)$.

Observe that if $\{q=0\}$ is a conic in $P_{C}^{2}$ and $T, Q, R, S$ are four points on $\{q=0\}$ with $\{T, Q\} \neq\{R, S\}$, then a quartic $C$ is such that $C \cap\{q=0\}=3 R+$ $3 S+T+Q$ if and only if $C$ is $\left\{r_{1} r_{2}^{3}+q g=0\right\}$ for a polynomial $g$ of degree 2 , where $\left\{r_{1}=0\right\}$ is the line $T Q$ and $\left\{r_{2}=0\right\}$ is the line $R S$. Besides in this case $\left\{r_{1}=0\right\}$ is bitangent to $C$ in $T$ and $Q$ if and only if $T=Q$ or $g(T)=g(Q)=0$. Thus, "forgetting $v$ ", $L$ is not normally generated if and only if a) and b) hold:

a) $C$ is a smooth curve of the following type: choose a conic $\{q=0\}$ in $\boldsymbol{P}_{\boldsymbol{C}}^{2}$ and four points on $\{q=0\}, T, Q, R, S$, with $\{T, Q\} \neq\{R, S\}$; let the line $T Q$ be $\left\{r_{1}=0\right\}$ and the line $R S$ be $\left\{r_{2}=0\right\}$; choose a polynomial $g$ of degree 2 such that, if $T \neq Q, g(T)=g(Q)=0$; let $C$ be the curve $\left\{r_{1} r_{2}^{3}+q g=0\right\}$.

b) $z=\mu(T+Q)-\mu(R+S)(T, Q, R, S$ in a $)$. 
2. Case $M$ is of type $\left(1, d_{2}, d_{3}\right), d_{2} \geq 2$.

2a. Let $(Y, P)=\left(E_{1}, P_{1}\right) \times\left(E_{2}, P_{2}\right) \times\left(E_{3}, P_{3}\right), E_{l}$ elliptic curves, $z=\left(z_{1}, z_{2}, z_{3}\right) \in$ $E_{1} \times E_{2} \times E_{3}, w=\left(w_{1}, w_{2}, w_{3}\right) \in E_{1} \times E_{2} \times E_{3}$. We can suppose $P_{l}=(0)$.

We have to see whether in

$$
\bigcap_{n, m}\left(\left(\{0\} \times E_{2} \times E_{3}\right) \cup\left(E_{1} \times\{0\} \times E_{3}\right) \cup\left(E_{1} \times E_{2} \times\{0\}\right)\right)_{n z+m w}
$$

there is a point $b$ such that $2 b \in\langle z\rangle \oplus\langle w\rangle$.

- Suppose first $z_{l} \neq 0 \forall i$.

Let $d_{3}>3$; if $\left\{\text { ord } z_{l}\right\}_{l=1,2,3} \neq\{2,4,4\}$ and $\neq\{2,2, l\}$ for any $l \in N$, then

$$
\bigcap_{n}\left(\left(\{0\} \times E_{2} \times E_{3}\right) \cup\left(E_{1} \times\{0\} \times E_{3}\right) \cup\left(E_{1} \times E_{2} \times\{0\}\right)\right)_{n z}=\emptyset
$$

and thus

$$
\bigcap_{n, m}\left(\left(\{0\} \times E_{2} \times E_{3}\right) \cup\left(E_{1} \times\{0\} \times E_{3}\right) \cup\left(E_{1} \times E_{2} \times\{0\}\right)\right)_{n z+m w}=\emptyset ;
$$

if $\left\{\operatorname{ord} z_{l}\right\}_{l=1,2,3}=\{2,4,4\}$, for instance $\left(\operatorname{ord} z_{1}\right.$, ord $z_{2}$, ord $\left.z_{3}\right)=(2,4,4)$, then

$$
\bigcap_{n}\left(\left(\{0\} \times E_{2} \times E_{3}\right) \cup\left(E_{1} \times\{0\} \times E_{3}\right) \cup\left(E_{1} \times E_{2} \times\{0\}\right)\right)_{n z}=\left(0, z_{2}, 3 z_{3}\right)+\langle z\rangle
$$

and $\bigcap_{m}\left(\left(0, z_{2}, 3 z_{3}\right)+\langle z\rangle\right)_{m w} \neq \emptyset$ implies $w \in\langle z\rangle$ but this is absurd, thus

$$
\bigcap_{n, m}\left(\left(\{0\} \times E_{2} \times E_{3}\right) \cup\left(E_{1} \times\{0\} \times E_{3}\right) \cup\left(E_{1} \times E_{2} \times\{0\}\right)\right)_{n z+m w}=\emptyset ;
$$

if $\left\{\operatorname{ord} z_{l}\right\}_{l=1,2,3}=\{2,2, l\}$ with $l \geq 3$, for instance (ord $z_{1}$, ord $z_{2}$, ord $\left.z_{3}\right)=(2,2, l)$ with $l \geq 3$, then $\bigcap_{n}\left(\left(\{0\} \times E_{2} \times E_{3}\right) \cup\left(E_{1} \times\{0\} \times E_{3}\right) \cup\left(E_{1} \times E_{2} \times\{0\}\right)\right)_{n z}$ is equal to

$$
\left(\{0\} \times\left\{z_{2}\right\} \times E_{3}\right) \cup\left(\left\{z_{1}\right\} \times\{0\} \times E_{3}\right)
$$

and $\bigcap_{m}\left(\left(\{0\} \times\left\{z_{2}\right\} \times E_{3}\right) \cup\left(\left\{z_{1}\right\} \times\{0\} \times E_{3}\right)\right)_{m w} \neq \emptyset$ implies $w=z$ and this is absurd, thus

$$
\bigcap_{n, m}\left(\left(\{0\} \times E_{2} \times E_{3}\right) \cup\left(E_{1} \times\{0\} \times E_{3}\right) \cup\left(E_{1} \times E_{2} \times\{0\}\right)\right)_{n z+m w}=\emptyset .
$$

Thus if $d_{3}>3$ then $M$ is globally generated and $L$ normally generated.

Let $d_{3}=3$. Then $\bigcap_{n}\left(\left(\{0\} \times E_{2} \times E_{3}\right) \cup\left(E_{1} \times\{0\} \times E_{3}\right) \cup\left(E_{1} \times E_{2} \times\{0\}\right)\right)_{n z}$ is equal to

$$
\bigcup_{\sigma \in S_{3}}\left(\sigma(1) z_{1}, \sigma(2) z_{2}, \sigma(3) z_{3}\right)
$$

First we see when there is a point $b$ in $\bigcap_{m}\left(\bigcup_{\sigma \in S_{3}}\left(\sigma(1) z_{1}, \sigma(2) z_{2}, \sigma(3) z_{3}\right)\right)_{m w}$. 
Observe that $\bigcap_{m}\left(\bigcup_{\sigma \in S_{3}}\left(\sigma(1) z_{1}, \sigma(2) z_{2}, \sigma(3) z_{3}\right)\right)_{m w} \neq \emptyset$ if and only if $\left(\sigma(1) z_{1}, \sigma(2) z_{2}, \sigma(3) z_{3}\right)=\left(\tau(1) z_{1}, \tau(2) z_{2}, \tau(3) z_{3}\right)+w=\left(\gamma(1) z_{1}, \gamma(2) z_{2}, \gamma(3) z_{3}\right)+2 w$

for some $\sigma, \tau, \gamma \in S_{3}$. This is true if and only if $\gamma(i)-\tau(i)=\tau(i)-\sigma(i)$ for $i=1,2,3$, that is $2 \tau(i)=\sigma(i)+\gamma(i)$ for $i=1,2,3$. Suppose $(\tau(1), \tau(2), \tau(3))=$ $(0,1,2)$, thus $2(\tau(1), \tau(2), \tau(3))=(0,2,1)$. Observing first that $\sigma(i) \neq \gamma(i)$ for $i=1,2,3$, we see that $(\sigma(1), \sigma(2), \sigma(3))=(1,0,2)$ and $(\gamma(1), \gamma(2), \gamma(3))=(2,1,0)$ or the contrary. Thus $M$ is not globally generated if and only if $w=$ $\left(\varepsilon_{1} z_{1}, \varepsilon_{2} z_{2}, \varepsilon_{3} z_{3}\right)$ with $\left\{\varepsilon_{1}, \varepsilon_{2}, \varepsilon_{3}\right\}=\{0,1,-1\}$. Observe that in this case $2\left(\sigma(1) z_{1}\right.$, $\left.\sigma(2) z_{2}, \sigma(3) z_{3}\right) \in\langle z\rangle \oplus\langle w\rangle$. Thus $L$ is not normally generated if and only if $w=\left(\varepsilon_{1} z_{1}, \varepsilon_{2} z_{2}, \varepsilon_{3} z_{3}\right)$ with $\left\{\varepsilon_{1}, \varepsilon_{2}, \varepsilon_{3}\right\}=\{0,1,-1\}$.

- Suppose only one of the $z_{l}$ 's is equal to 0 , for instance $z_{3}=0$ and $z_{1} \neq 0$, $z_{2} \neq 0$.

If $\left\{\operatorname{ord}\left(z_{1}\right), \operatorname{ord}\left(z_{2}\right)\right\} \neq\{2,3\}$ then $\bigcap_{n, m}\left(\left(\{0\} \times E_{2} \times E_{3}\right) \cup\left(E_{1} \times\{0\} \times E_{3}\right) \cup\right.$ $\left.\left(E_{1} \times E_{2} \times\{0\}\right)\right)_{n z+m w}=\bigcap_{m}\left(E_{1} \times E_{2} \times\{0\}\right)_{m w}=\bigcap_{m}\left(E_{1} \times E_{2} \times\left\{m w_{3}\right\}\right) ;$ if $w_{3} \neq 0$ this set is empty and then $M$ is globally generated and $L$ normally generated; while if $w_{3}=0$ this set is equal $E_{1} \times E_{2} \times\{0\}$ and obviously $L$ is not normally generated. 3.

Now suppose $\left\{\operatorname{ord}\left(z_{1}\right), \operatorname{ord}\left(z_{2}\right)\right\}=\{2,3\} ;$ for instance $\operatorname{ord}\left(z_{1}\right)=2, \operatorname{ord}\left(z_{2}\right)=$

Then $\bigcap_{n}\left(\left(\{0\} \times E_{2} \times E_{3}\right) \cup\left(E_{1} \times\{0\} \times E_{3}\right) \cup\left(E_{1} \times E_{2} \times\{0\}\right)\right)_{n z}=\left(\{0\} \times\left\{z_{2}\right\}\right.$ $\left.\times E_{3}\right) \cup\left(E_{1} \times E_{2} \times\{0\}\right)$. It is possible that $\bigcap_{m}\left(\left(\{0\} \times\left\{z_{2}\right\} \times E_{3}\right) \cup\left(E_{1} \times E_{2} \times\right.\right.$ $\{0\}))_{m w} \neq \emptyset$ if and only if we are in one of the following five cases:

i) $w_{3}=0$

ii) $w_{1}=0$ and $w_{2}=0$,

iii) $\operatorname{ord}\left(w_{3}\right)=2$ and $\left\{\operatorname{ord}\left(w_{1}\right), \operatorname{ord}\left(w_{2}\right)\right\} \subseteq\{1,3\}$,

iv) $\operatorname{ord}\left(w_{3}\right)=3$ and $\left\{\operatorname{ord}\left(w_{1}\right)\right.$, ord $\left.\left(w_{2}\right)\right\} \subseteq\{1,2\}$,

v) $\left\{\operatorname{ord}\left(w_{1}\right), \operatorname{ord}\left(w_{2}\right), \operatorname{ord}\left(w_{3}\right)\right\} \subseteq\{1,2\}$.

In these cases $L$ is not normally generated, in fact one easily sees that there is a point $b \in \bigcap_{m}\left(\left(\{0\} \times\left\{z_{2}\right\} \times E_{3}\right) \cup\left(E_{1} \times E_{2} \times\{0\}\right)\right)_{m w}$ such that $2 b \in\langle z\rangle \oplus$ $\langle w\rangle$ : in the first two cases it is obvious, in the third one we can take $b=\left(w_{1}, z_{2}+w_{2}, 0\right)$, in the forth case we can take $b=\left(0, z_{2}, w_{3}\right)$ and in the last case we can take $b=\left(0, z_{2}, w_{3}\right)$ or $b=\left(w_{1}, z_{2}+w_{2}, 0\right)$.

- Suppose only two of the $z_{l}$ 's are equal to 0 , for instance $z_{2}=0$ and $z_{3}=0$ and $z_{1} \neq 0$.

Then $\bigcap_{n}\left(\left(\{0\} \times E_{2} \times E_{3}\right) \cup\left(E_{1} \times\{0\} \times E_{3}\right) \cup\left(E_{1} \times E_{2} \times\{0\}\right)\right)_{n z}$ is equal to $\left(E_{1} \times\{0\} \times E_{3}\right) \cup\left(E_{1} \times E_{2} \times\{0\}\right)$.

It is possible that $\bigcap_{m}\left(\left(E_{1} \times\{0\} \times E_{3}\right) \cup\left(E_{1} \times E_{2} \times\{0\}\right)\right)_{m w} \neq \emptyset$ if and only if we are in one of the following four cases:

i) $w_{3}=0$,

ii) $w_{2}=0$,

iii) $\left\{\operatorname{ord}\left(w_{2}\right), \operatorname{ord}\left(w_{3}\right)\right\} \subseteq\{1,2\}$,

iv) $\left\{\operatorname{ord}\left(w_{2}\right)\right.$, ord $\left.\left(w_{3}\right)\right\} \subseteq\{2,3\}$. 
In these cases $L$ is not normally generated, in fact one easily sees that there is a point $b \in \bigcap_{m}\left(\left(E_{1} \times\{0\} \times E_{3}\right) \cup\left(E_{1} \times E_{2} \times\{0\}\right)\right)_{m w}$ such that $2 b \in$ $\langle z\rangle \oplus\langle w\rangle$; in the first two cases it is obvious, in the third one the set $\bigcap_{m}\left(\left(E_{1} \times\{0\} \times E_{3}\right) \cup\left(E_{1} \times E_{2} \times\{0\}\right)\right)_{m w}$ contains $E_{1} \times\left\{w_{2}\right\} \times\{0\}$ and $E_{1} \times\{0\} \times\left\{w_{3}\right\}$, in the forth case, if for instance $\operatorname{ord}\left(w_{2}\right)=2$ and $\operatorname{ord}\left(w_{3}\right)=3$, the set $\bigcap_{m}\left(\left(E_{1} \times\{0\} \times E_{3}\right) \cup\left(E_{1} \times E_{2} \times\{0\}\right)\right)_{m w}$ contains $E_{1} \times\{0\} \times\left\{w_{3}\right\}$.

2b. Let $(Y, P)=(J(C), \Theta) \times\left(E, P^{\prime}\right), E$ elliptic curve, $C$ Riemann surface of genus $2, z=\left(z_{1}, z_{2}\right) \in J(C) \times E, w=\left(w_{1}, w_{2}\right) \in J(C) \times E$.

Let $\left(Y^{\prime}, N\right)$ be the abelian variety and the line bundle that arises from $(Y, P)$ and from the subgroup $\langle z\rangle$.

If we are in one of the following three cases

i) $\operatorname{ord}\left(z_{1}\right) \geq 5$

ii) $\operatorname{ord}\left(z_{1}\right)=3$ and $\operatorname{ord}\left(z_{2}\right)=2$ or $>3$

iii) $\operatorname{ord}\left(z_{1}\right)=4$ and $\operatorname{ord}\left(z_{2}\right)=3$ or $\geq 5$,

then $N$ is globally generated (we see this in case 1) and then $M$ is globally generated (as observed in [B-L-R] Remark 1.5) and thus $L$ is normally generated.

Thus it remains to consider the following four cases:

i) $\operatorname{ord}\left(z_{1}\right)=2$ and $\operatorname{ord}\left(z_{2}\right)>2$,

ii) $\operatorname{ord}\left(z_{1}\right)=4$ and $\operatorname{ord}\left(z_{2}\right)=2$,

iii) $\operatorname{ord}\left(z_{1}\right)=\operatorname{ord}\left(z_{2}\right)=4$,

iv) $\operatorname{ord}\left(z_{1}\right)=\operatorname{ord}\left(z_{2}\right)=3$.

- $\operatorname{ord}\left(z_{1}\right)=2$ and $\operatorname{ord}\left(z_{2}\right)>2$.

Observe that

$$
\begin{aligned}
\bigcap_{n, m}\left(\left(V^{1} \times E\right) \cup(J(C) \times\{0\})\right)_{n z+m w} \\
=\bigcap_{m}\left(\left(V^{1} \cap V_{z_{1}}^{1}\right) \times E\right)_{m w} \\
=\left(\bigcap_{m}\left(V^{1} \cap V_{z_{1}}^{1}\right)_{m w_{1}}\right) \times E
\end{aligned}
$$

If $w_{1} \neq 0$ then $\bigcap_{m}\left(V^{1} \cap V_{z_{1}}^{1}\right)_{m w_{1}}=\emptyset$, in fact, if it contains a point then it must contain at least 4 points because it is invariant for $\left\langle w_{1}\right\rangle+\left\langle z_{1}\right\rangle$ and on the other hand it contains at most 2 points. Thus $M$ is globally generated and $L$ normally generated.

Let $w_{1}=0$; we have already seen that $\exists b \in\left(V^{1} \cap V_{z_{1}}^{1}\right) \times E$ such that $2 b \in Z$, in fact $\exists a \in V^{1} \cap V_{z_{1}}^{1}$ such that $2 a \in\left\langle z_{1}\right\rangle$ : since $2 z_{1}=0$ one can write $z_{1}=$ $\mu(R)-\mu(S)$ with $R$ and $S$ in the set of the points that are fixed by the hyperelliptic involution of $C$; thus we can take $a=\mu(R)$ because $\mu(R) \in V^{1} \cap V_{z_{1}}^{1}$ and $2 \mu(R)=0$. Thus $L$ is never normally generated.

- $\operatorname{ord}\left(z_{1}\right)=4$ and $\operatorname{ord}\left(z_{2}\right)=2$.

Observe that 


$$
\begin{aligned}
\bigcap_{n, m}\left(\left(V^{1} \times E\right) \cup(J(C) \times\{0\})\right)_{n z+m w} \\
\quad=\bigcap_{m}\left(\left(\left(V^{1} \cap V_{2 z_{1}}^{1}\right) \times\left\{z_{2}\right\}\right) \cup\left(\left(V_{z_{1}}^{1} \cap V_{3 z_{1}}^{1}\right) \times\{0\}\right)\right)_{m w} .
\end{aligned}
$$

If $w_{2}=0$ this set is equal to

$$
\left(\left(\bigcap_{m}\left(V^{1} \cap V_{2 z_{1}}^{1}\right)_{m w_{1}}\right) \times\left\{z_{2}\right\}\right) \cup\left(\left(\bigcap_{m}\left(V_{z_{1}}^{1} \cap V_{3 z_{1}}^{1}\right)_{m w_{1}}\right) \times\{0\}\right) .
$$

Thus if $w_{1} \neq 2 z_{1}$ this set is empty (because $\bigcap_{m}\left(V^{1} \cap V_{2 z_{1}}^{1}\right)_{m w_{1}}$ is invariant for $\left.\left\langle 2 z_{1}\right\rangle+\left\langle w_{1}\right\rangle \cdots\right)$, thus $M$ is globally generated and $L$ is normally generated. While, if $w_{1}=2 z_{1}$, then $L$ is never normally generated (in fact $\exists b \in$ $\left(V^{1} \cap V_{2 z_{1}}^{1}\right) \times E$ such that $2 b \in Z$, in fact $\exists a \in V^{1} \cap V_{2 z_{1}}^{1}$ such that $2 a=0$ : since $4 z_{1}=0$ one can write $2 z_{1}=\mu(R)-\mu(S)$ with $R$ and $S$ in the set of the points that are fixed by the hyperelliptic involution of $C$; thus we can take $a=\mu(R)$ because $\mu(R) \in V^{1} \cap V_{2 z_{1}}^{1}$ and $2 \mu(R)=0$.)

Now let $w_{2} \neq 0$. Call $A_{m}=\left(\left(\left(V^{1} \cap V_{2 z_{1}}^{1}\right) \times\left\{z_{2}\right\}\right) \cup\left(\left(V_{z_{1}}^{1} \cap V_{3 z_{1}}^{1}\right) \times\{0\}\right)\right)_{m w}$. Consider for instance the intersection of the first piece of $A_{0}$ with the two pieces of $A_{1}$; we obtain

$$
\begin{aligned}
& \left(\left(\left(V^{1} \cap V_{2 z_{1}}^{1}\right) \times\left\{z_{2}\right\}\right) \cap\left(\left(V_{w_{1}}^{1} \cap V_{2 z_{1}+w_{1}}^{1}\right) \times\left\{z_{2}+w_{2}\right\}\right)\right) \\
& \quad \cup\left(\left(\left(V^{1} \cap V_{2 z_{1}}^{1}\right) \times\left\{z_{2}\right\}\right) \cap\left(\left(V_{z_{1}+w_{1}}^{1} \cap V_{3 z_{1}+w_{1}}^{1}\right) \times\left\{w_{2}\right\}\right)\right) .
\end{aligned}
$$

If $w_{2} \neq z_{2}$ this set is empty. If $w_{2}=z_{2}$ we obtain $\left(\left(V^{1} \cap V_{2 z_{1}}^{1}\right) \cap\left(V^{1} \cap V_{2 z_{1}}^{1}\right)_{z_{1}+w_{1}}\right)$ $\times\left\{z_{2}\right\}$; suppose $V^{1} \cap V_{2 z_{1}}^{1}=\left\{a, a+2 z_{1}\right\}$ and then $\left(V^{1} \cap V_{2 z_{1}}^{1}\right)_{z_{1}+w_{1}}=\left\{a+z_{1}+w_{1}\right.$, $\left.a+2 z_{1}+z_{1}+w_{1}\right\}$; thus it is necessary for $\left(V^{1} \cap V_{2 z_{1}}^{1}\right) \cap\left(V^{1} \cap V_{2 z_{1}}^{1}\right)_{z_{1}+w_{1}} \neq \emptyset$ that $z_{1}=w_{1}$ or $z_{1}=-w_{1}$ or $3 z_{1}=-w_{1}$ and this is impossible since $\langle z\rangle \neq\langle w\rangle$. Thus if $w_{2} \neq 0$ then $M$ is globally generated and $L$ is normally generated.

- $\operatorname{ord}\left(z_{1}\right)=\operatorname{ord}\left(z_{2}\right)=4$.

Since ord $(w) \mid \operatorname{ord}(z)=4$, ord $(w)$ must be 2 or 4 . Observe that

$$
\begin{aligned}
\bigcap_{n, m}\left(\left(V^{1} \times E\right) \cup(J(C) \times\{0\})\right)_{n z+m w} & =\bigcap_{m}\left(\bigcap_{n}\left(\left(V^{1} \times E\right) \cup(J(C) \times\{0\})\right)_{n z}\right)_{m w} \\
& =\bigcap_{m} B_{m w},
\end{aligned}
$$

where $B=\left(\left(V^{1} \cap V_{z_{1}}^{1} \cap V_{2 z_{1}}^{1}\right) \times\left\{3 z_{2}\right\}\right) \cup\left(\left(V^{1} \cap V_{z_{1}}^{1} \cap V_{3 z 1}^{1}\right) \times\left\{2 z_{2}\right\}\right) \cup\left(\left(V_{z_{1}}^{1} \cap V_{2 z_{1}}^{1} \cap\right.\right.$ $\left.\left.V_{3 z_{1}}^{1}\right) \times\{0\}\right)$. If $w_{2}=0$ we have that $\bigcap_{m} B_{m w}$ is equal to

$$
\begin{aligned}
& \left(\bigcap_{m}\left(V^{1} \cap V_{z_{1}}^{1} \cap V_{2 z_{1}}^{1}\right)_{m w_{1}} \times\left\{3 z_{2}\right\}\right) \cup\left(\bigcap_{m}\left(V^{1} \cap V_{z_{1}}^{1} \cap V_{3 z_{1}}^{1}\right)_{m w_{1}} \times\left\{2 z_{2}\right\}\right) \\
& \cup\left(\bigcap_{m}\left(V_{z_{1}}^{1} \cap V_{2 z_{1}}^{1} \cap V_{3 z_{1}}^{1}\right)_{m w_{1}} \times\{0\}\right) .
\end{aligned}
$$


Consider $\bigcap_{m}\left(V^{1} \cap V_{z_{1}}^{1} \cap V_{2 z_{1}}^{1}\right)_{m w_{1}}$; it is invariant for $w_{1}$. If $\operatorname{ord}\left(w_{1}\right)=4$ if it contains a point it must contain 4 points and this is absurd, thus it is empty. If $\operatorname{ord}\left(w_{1}\right)=2$, let $V^{1} \cap V_{z_{1}}^{1}=\left\{a_{1}, a_{2}\right\}$ and then $V_{z_{1}}^{1} \cap V_{2 z_{1}}^{1}=\left\{a_{1}+z_{1}, a_{2}+z_{1}\right\}$; it is necessary for $V^{1} \cap V_{z_{1}}^{1} \cap V_{2 z_{1}}^{1} \neq \emptyset$ that $a_{2}=a_{1} \pm z_{1}$; thus, if $\bigcap_{m}\left(V^{1} \cap V_{z_{1}}^{1}\right.$ $\left.\cap V_{2 z_{1}}^{1}\right)_{m w_{1}} \neq \emptyset$ then $a_{1}$ (and $a_{2}$ ) must be invariant for $w_{1}$ and this is absurd. Analogously the other pieces. Thus $M$ is globally generated and $L$ is normally generated.

Suppose now $w_{2} \neq 0$. If $\operatorname{ord}\left(w_{2}\right)=4$ then $\operatorname{ord}(w)=4$ and thus $\bigcap_{m} B_{m w}=$

$\emptyset$. Suppose $\operatorname{ord}\left(w_{2}\right)=2$; if $\operatorname{ord}\left(w_{1}\right)=4$ we have immediately that $\bigcap_{m}^{m} B_{m w}=$ $\emptyset$ because for instance $\left(V^{1} \cap V_{z_{1}}^{1} \cap V_{2 z_{1}}^{1}\right) \cap\left(V^{1} \cap V_{z_{1}}^{1} \cap V_{2 z_{1}}^{1}\right)_{2 w_{1}}=\emptyset$; if ord $\left(w_{1}\right)=$ 2 it is necessary for $\bigcap_{m} B_{m w} \neq \emptyset$ that $w_{2}=z_{2}$ or $w_{2}=3 z_{2}$ and this is absurd because $\operatorname{ord}\left(w_{2}\right)=2$ while $\operatorname{ord}\left(z_{2}\right)=4$; thus $M$ is globally generated and $L$ is normally generated.

- $\operatorname{ord}\left(z_{1}\right)=\operatorname{ord}\left(z_{2}\right)=3$.

Since $\operatorname{ord}(w) \mid \operatorname{ord}(z)=3, \operatorname{ord}(w)=3$. Observe that

$$
\begin{aligned}
\bigcap_{n, m}\left(\left(V^{1} \times E\right) \cup(J(C) \times\{0\})\right)_{n z+m w} \\
\quad=\bigcap_{m}\left(\bigcap_{n}\left(\left(V^{1} \times E\right) \cup(J(C) \times\{0\})\right)_{n z}\right)_{m w} \\
\quad=\bigcap_{m}\left(\left(\left(V^{1} \cap V_{z_{1}}^{1}\right) \times\left\{2 z_{2}\right\}\right) \cup\left(\left(V^{1} \cap V_{2 z_{1}}^{1}\right) \times\left\{z_{2}\right\}\right) \cup\left(\left(V_{z_{1}}^{1} \cap V_{2 z_{1}}^{1}\right) \times\{0\}\right)\right)_{m w} .
\end{aligned}
$$

If $w_{2}=0$ this set is

$$
\begin{aligned}
& \left(\left(V^{1} \cap V_{z_{1}}^{1}\right) \cap\left(V^{1} \cap V_{z_{1}}^{1}\right)_{w_{1}} \cap\left(V^{1} \cap V_{z_{1}}^{1}\right)_{2 w_{1}}\right) \times\left\{2 z_{2}\right\} \\
& U\left(\left(V^{1} \cap V_{2 z_{1}}^{1}\right) \cap\left(V^{1} \cap V_{2 z_{1}}^{1}\right)_{w_{1}} \cap\left(V^{1} \cap V_{2 z_{1}}^{1}\right)_{2 w_{1}}\right) \times\left\{z_{2}\right\} \\
& U\left(\left(V_{2 z_{1}}^{1} \cap V_{z_{1}}^{1}\right) \cap\left(V_{2 z_{1}}^{1} \cap V_{z_{1}}^{1}\right)_{w_{1}} \cap\left(V_{2 z_{1}}^{1} \cap V_{z_{1}}^{1}\right)_{2 w_{1}}\right) \times\{0\}
\end{aligned}
$$

and this is empty because $\left(V^{1} \cap V_{z_{1}}^{1}\right) \cap\left(V^{1} \cap V_{z_{1}}^{1}\right)_{w_{1}} \cap\left(V^{1} \cap V_{z_{1}}^{1}\right)_{2 w_{1}}$ is invariant for $w_{1}$ thus if it contains a point then it must contain at least three points and this is absurd. Thus $M$ is globally generated and $L$ is normally generated.

Suppose $w_{2} \neq 0$. It is necessary for

$$
\begin{gathered}
\bigcap_{m}\left(\left(\left(V^{1} \cap V_{z_{1}}^{1}\right) \times\left\{2 z_{2}\right\}\right) \cup\left(\left(V^{1} \cap V_{2 z_{1}}^{1}\right) \times\left\{z_{2}\right\}\right) \cup\left(\left(V_{z_{1}}^{1} \cup V_{2 z_{1}}^{1}\right) \times\{0\}\right)\right)_{m w} \neq \emptyset \text { that } \\
w_{2}=2 z_{2} \text { and }\left(V^{1} \cap V_{z_{1}}^{1}\right) \cap\left(V^{1} \cap V_{2 z_{1}}^{1}\right)_{w_{1}} \cap\left(V_{z_{1}}^{1} \cap V_{2 z_{1}}^{1}\right)_{2 w_{1}} \neq \emptyset
\end{gathered}
$$

or

$$
w_{2}=z_{2} \quad \text { and } \quad\left(V^{1} \cap V_{z_{1}}^{1}\right) \cap\left(V_{z_{1}}^{1} \cap V_{2 z_{1}}^{1}\right)_{w_{1}} \cap\left(V^{1} \cap V_{2 z_{1}}^{1}\right)_{2 w_{1}} \neq \emptyset ;
$$

but both cases are impossible, for instance consider the first one: if $w_{1}=$ $0\left(V^{1} \cap V_{z_{1}}^{1}\right) \cap\left(V^{1} \cap V_{2 z_{1}}^{1}\right)_{w_{1}} \cap\left(V_{z_{1}}^{1} \cap V_{2 z_{1}}^{1}\right)_{2 w_{1}}=V^{1} \cap V_{z_{1}}^{1} \cap V_{2 z_{1}}^{1}=\emptyset$, if $w_{1} \neq 0$ the 
set $\left(V^{1} \cap V_{z_{1}}^{1}\right) \cap\left(V^{1} \cap V_{2 z_{1}}^{1}\right)_{w_{1}} \cap\left(V_{z_{1}}^{1} \cap V_{2 z_{1}}^{1}\right)_{2 w_{1}}$ is invariant for $w_{1}$, thus if it contains a point then it must contain at least 3 points and this is absurd. Thus $M$ is globally generated and $L$ is normally generated.

2c. Let $(Y, P)=(J(C), \Theta)$ with $C$ not hyperelliptic Riemann surface of genus 3. Let $d_{3} \geq 5$. Let $\left(Y^{\prime}, N\right)$ be the abelian variety and the line bundle that arises from $(Y, P)$ and from the subgroup $\langle z\rangle$. Then $N$ is globally generated by Theorem 1a in [B-L-R] and then $M$ is globally generated (as observed in [B-L-R] Remark 1.5) and thus $L$ is normally generated.

Q.E.D.

Remark 1. Theorem 1 characterizes normally generated line bundles on abelian threefolds except for the case $(Y, P)=(J(C), \Theta)$ with $C$ hyperelliptic Riemann surface of genus 3 and for the cases $(Y, P)=(J(C), \Theta)$ with $C$ not hyperelliptic Riemann surface of genus 3 and $M$ of type $(1,1,4)$ or of type $\left(1, d_{2}, d_{3}\right)$ with $d_{2} \geq 2$ and $d_{3}<5$.

If $(Y, P)=(J(C), \Theta)$ with $C$ hyperelliptic Riemann surface of genus 3 and $M$ is of type $(1,1,3)$ one can prove that generically $L$ is normally generated.

In fact:

Let $C$ be the curve $y^{2}=p(x), p(x)$ polynomial of degree 8 without multiple roots; we see $C$ as the Riemann surface obtained by glueing two copies of $\boldsymbol{P}^{1}$ as described for instance in [F-R] p. 145; let $P_{1}^{\infty}$ and $P_{2}^{\infty}$ be the two points at infinity in the two copies of $\boldsymbol{P}^{1}$.

$L$ is not normally generated if and only if $\exists b=\mu\left(P_{0}+Q_{0}\right)=\mu\left(P_{1}+Q_{1}\right)+z$ $=\mu\left(P_{2}+Q_{2}\right)+2 z \in V^{2} \cap V_{z}^{2} \cap V_{2 z}^{2}$ such that $2 b \in\langle z\rangle+\mu\left(K_{C}\right)$.

We recall that $K_{C}=2 g_{2}^{1}$ where $g_{2}^{1}$ is the linear system of degree 2 and of dimension 1 on $C$. Besides observe that $\mathscr{L}\left(2 P_{1}^{\infty}+2 P_{2}^{\infty}\right)=\left\langle 1, x, x^{2}\right\rangle$, thus the divisors given by the sections of $K_{C}\left(\ni 2 P_{1}^{\infty}+2 P_{2}^{\infty}\right)$ are invariant for the hyperelliptic involution.

One can suppose $2 b=\mu\left(K_{C}\right)$. Observe that it is not possible that $P_{0}=$ $\sigma\left(Q_{0}\right)$ where $\sigma$ is the hyperelliptic involution; in fact in this case $\mu\left(P_{0}+Q_{0}\right)=0$, then $\mu\left(P_{2}+Q_{2}\right)=z$, then $3 \mu\left(P_{2}+Q_{2}\right)=0$, then $3\left(P_{2}+Q_{2}\right) \sim 3 P_{1}^{\infty}+3 P_{2}^{\infty}$; but $\mathscr{L}\left(3 P_{1}^{\infty}+3 P_{2}^{\infty}\right)=\left\langle 1, x, x^{2}, x^{3}\right\rangle$, thus this implies that $P_{2}=\sigma\left(Q_{2}\right)$ and then $z=0$, which is absurd.

Thus $L$ is normally generated if and only if $\exists P_{l}, Q_{i} i=1,2,3$ such that $\mu\left(P_{0}+Q_{0}\right)=\mu\left(P_{1}+Q_{1}\right)+z=\mu\left(P_{2}+Q_{2}\right)+2 z$ and $P_{0}=\sigma\left(P_{0}\right)$ and $Q_{0}=\sigma\left(Q_{0}\right)$.

Observe this is equivalent to: $\exists P_{l}, Q_{i} i=1,2,3$ such that $\mu\left(P_{0}+Q_{0}\right)-\mu$ $\left(P_{1}+Q_{1}\right)=z=2 \mu\left(P_{1}+Q_{1}\right), P_{0}=\sigma\left(P_{0}\right), Q_{0}=\sigma\left(Q_{0}\right), P_{2}=\sigma\left(P_{1}\right), Q_{2}=\sigma\left(Q_{1}\right)$ (modulo changing $P$ with $Q$ ). This is equivalent to: $\exists P_{l}, Q_{i} i=1,2$ such that $P_{0}=\sigma\left(P_{0}\right), Q_{0}=\sigma\left(Q_{0}\right)$,

$$
\begin{gathered}
6\left(P_{1}+Q_{1}\right) \in 3 K_{C} \\
P_{0}+Q_{0}+3\left(P_{1}+Q_{1}\right) \in 2 K_{C}
\end{gathered}
$$

and $z=2 \mu\left(P_{1}+Q_{1}\right)$.

Observe that

$$
\mathscr{L}\left(4 P_{1}^{\infty}+4 P_{2}^{\infty}\right)=\left\langle 1, x, x^{2}, x^{3}, x^{4}, y\right\rangle
$$


and

$$
\mathscr{L}\left(6 P_{1}^{\infty}+6 P_{2}^{\infty}\right)=\left\langle 1, x, x^{2}, x^{3}, x^{4}, x^{5}, x^{6}, y, y x, y x^{2}\right\rangle .
$$

Thus (1) is equivalent to say that there are a polynomial of degree $\leq 2, r(x)$, and a polynomial of degree $\leq 6, q(x)$, such that the divisor given by $y r(x)+q(x)$ on $C$ is $6 P_{1}+6 Q_{1}$ that is

$$
q^{2}(x)-p(x) r^{2}(x)=\left(x-x_{P_{1}}\right)^{6}\left(x-x_{Q_{1}}\right)^{6},
$$

and (2) is equivalent to say that there are a polynomial of degree $\leq 4, s(x)$, such that the divisor given by $y-s(x)$ on $C$ is $3 P_{1}+3 Q_{1}+P_{0}+Q_{0}$ that is

$$
s^{2}(x)-p(x)=\left(x-x_{P_{0}}\right)\left(x-x_{Q_{0}}\right)\left(x-x_{P_{1}}\right)^{3}\left(x-x_{Q_{1}}\right)^{3},
$$

that is

$$
p(x)=s^{2}(x)-\left(x-x_{P_{0}}\right)\left(x-x_{Q_{0}}\right)\left(x-x_{P_{1}}\right)^{3}\left(x-x_{Q_{1}}\right)^{3} ;
$$

but, by a calculus of dimensions, there is a $p(x)$ of degree 8 without multiple roots that doesn't satisfy the last equation.

Proposition 1. (Small generalization of Birkenhake-Lange-Ramanan's Theorem on global generation of primitive lines bundles on abelian threefolds in [B-L-R]). Let $X$ be a complex torus of dimension three and $M$ an ample line bundle on $X$ of type $\left(1, d_{2}, d_{3}\right)$ such that $d_{3}>2$. Let $\pi:(X, M) \rightarrow(Y, P)$ be an isogeny with $\pi^{*} P=M,(Y, P)$ p.p.a.v. and such that $\operatorname{ker} \pi$ (and then $\operatorname{ker} \hat{\pi}$, where $\hat{\pi}: \hat{Y} \rightarrow \hat{X}$ is the dual isogeny) is isomorphic to $\mathbf{Z} / d_{2} \oplus \mathbf{Z} / d_{3}$. Let $Z:=\phi_{P}^{-1}$ $(\operatorname{ker} \hat{\pi}) \simeq \mathbf{Z} / d_{2} \oplus \mathbf{Z} / d_{3}$ in $Y$.

1. Suppose first that $d_{2}=1$. Let $Z=\langle z\rangle$.

1a. Let $(Y, P)=\left(E_{1}, P_{1}\right) \times\left(E_{2}, P_{2}\right) \times\left(E_{3}, P_{3}\right)$, $E_{l}$ elliptic curves, $z=\left(z_{1}, z_{2}, z_{3}\right) \in$ $E_{1} \times E_{2} \times E_{3}$. Then $M$ is globally generated if and only if $z_{l} \neq 0 \forall i, d_{3} \geq 4$ and $\left\{\right.$ ord $z_{1}$, ord $z_{2}$, ord $\left.z_{3}\right\} \neq\{2,4,4\}$ and $\neq\{2,2, l\}$ for any $l \in N$.

1b. Let $(Y, P)=(J(C), \Theta) \times\left(E, P^{\prime}\right), E$ elliptic curve, $C$ Riemann surface of genus $2, z=\left(z_{1}, z_{2}\right) \in J(C) \times E, z_{l} \neq 0 i=1,2$.

Then $M$ is globally generated if and only if we are not in one of the following four cases:

- $\operatorname{ord}\left(z_{1}\right)=2$

- $\operatorname{ord}\left(z_{1}\right)=\operatorname{ord}\left(z_{2}\right)=3$

- $\operatorname{ord}\left(z_{1}\right)=4$ and $\operatorname{ord}\left(z_{2}\right)=2$

- $\operatorname{ord}\left(z_{1}\right)=\operatorname{ord}\left(z_{2}\right)=4$ and $V^{1} \cap V_{z_{1}}^{1} \cap V_{2 z_{1}}^{1}=\emptyset$.

1c. Let $(Y, P)=(J(C), \Theta)$ with $C$ Riemann surface of genus 3 .

If $d_{3} \geq 5$ and $C$ is not hyperelliptic, $M$ is globally generated.

If $d_{3}=3$ then $M$ is not globally generated. 
2. Suppose now that $\left.d_{2}\right\rangle 1$. Let $Z=\langle w\rangle \oplus\langle z\rangle$ with $\operatorname{ord}(w)=d_{2}$ and $\operatorname{ord}(z)=$ $d_{3}$.

2a. Let $(Y, P)=\left(E_{1}, P_{1}\right) \times\left(E_{2}, P_{2}\right) \times\left(E_{3}, P_{3}\right), E_{l}$ elliptic curves $z=\left(z_{1}, z_{2}, z_{3}\right) \epsilon$ $E_{1} \times E_{2} \times E_{3}, w=\left(w_{1}, w_{2}, w_{3}\right) \in E_{1} \times E_{2} \times E_{3}$.

- Suppose $z_{l} \neq 0 \forall i$. If $d_{3}>3$ then $M$ is globally generated. If $d_{3}=3$ then $M$ is not globally generated if and only if $w=\left(\varepsilon_{1} z_{1}, \varepsilon_{2} z_{2}, \varepsilon_{3} z_{3}\right)$ with $\left\{\varepsilon_{1}, \varepsilon_{2}, \varepsilon_{3}\right\}=$ $\{0,1,-1\}$.

- Suppose only one of the $z_{l}$ 's is equal to 0 , for instance $z_{3}=0$ and $z_{1} \neq 0$, $z_{2} \neq 0$. Then $M$ is not globally generated if and only if we are in one of the following five cases:

i) $w_{3}=0$;

ii) $\left\{\operatorname{ord}\left(z_{1}\right), \operatorname{ord}\left(z_{2}\right)\right\}=\{2,3\}, w_{1}=0$ and $w_{2}=0$;

iii) $\left\{\operatorname{ord}\left(z_{1}\right), \operatorname{ord}\left(z_{2}\right)\right\}=\{2,3\}$, ord $\left(w_{3}\right)=2$ and $\left\{\operatorname{ord}\left(w_{1}\right)\right.$, ord $\left.\left(w_{2}\right)\right\} \subseteq\{1,3\}$;

iv) $\left\{\operatorname{ord}\left(z_{1}\right), \operatorname{ord}\left(z_{2}\right)\right\}=\{2,3\}$, ord $\left(w_{3}\right)=3$ and $\left\{\operatorname{ord}\left(w_{1}\right), \operatorname{ord}\left(w_{2}\right)\right\} \subseteq\{1,2\}$;

v) $\left\{\operatorname{ord}\left(z_{1}\right), \operatorname{ord}\left(z_{2}\right)\right\}=\{2,3\},\left\{\operatorname{ord}\left(w_{1}\right), \operatorname{ord}\left(w_{2}\right), \operatorname{ord}\left(w_{3}\right)\right\} \subseteq\{1,2\}$.

- Suppose only two of the $z_{l}$ 's are equal to 0 , for instance $z_{2}=0$ and $z_{3}=0$ and $z_{1} \neq 0$. Then $M$ is not globally generated if and only if we are in one of the following four cases:
i) $w_{3}=0$;
ii) $w_{2}=0$;
iii) $\left\{\operatorname{ord}\left(w_{2}\right)\right.$, ord $\left.\left(w_{3}\right)\right\} \subseteq\{1,2\}$;
iv) $\left\{\operatorname{ord}\left(w_{2}\right), \operatorname{ord}\left(w_{3}\right)\right\} \subseteq\{2,3\}$.

2b. Let $(Y, P)=(J(C), \Theta) \times\left(E, P^{\prime}\right), E$ elliptic curve, $C$ Riemann surface of genus $2, z=\left(z_{1}, z_{2}\right) \in J(C) \times E, w=\left(w_{1}, w_{2}\right) \in J(C) \times E$.

Then $M$ is not globally generated if and only if we are in one of the following two cases:

- $\operatorname{ord}\left(z_{1}\right)=2, \operatorname{ord}\left(z_{2}\right)>2$ and $w_{1}=0$

- $\operatorname{ord}\left(z_{1}\right)=4, \operatorname{ord}\left(z_{2}\right)=2, w_{2}=0$ and $w_{1}=2 z_{1}$.

2c. Let $(Y, P)=(J(C), \Theta)$ with $C$ not hyperelliptic Riemann surface of genus 3 . If $d_{3} \geq 5$ then $M$ is globally generated.

Remark 2. Let $(X, M)$ be an abelian variety with $M$ an ample line bundle of type $\left(1, d_{2}, \ldots, d_{g}\right)$ with $d_{g}>2$. One can conjecture that if $L=M^{2}$ is not normally generated then there exists an isogeny $(X, M) \rightarrow(Y, P)$ such that $\pi^{*} P=M, \operatorname{ker} \pi$ is isomorphic to $\bigoplus_{i=2, \ldots, g} \mathbf{Z} / d_{i}$ and $(Y, P)$ is a nontrivial product of p.p.a.v.'s. One can easily see that this conjecture is false: the dimension of every component of the moduli space of abelian varieties of fixed type $\left(2 d_{1}, \ldots, 2 d_{g}\right)$ with $d_{1} \cdot \ldots \cdot d_{g}=d$ and such that the line bundles inducing the polarizations are not normally generated is $\geq(g(g+1) / 2)-d$, thus it is $\geq 6-d$ if $g=3$, it is $\geq 10-d$ if $g=4$, it is $\geq 15-d$ if $g=5 \ldots$; while the dimension of every component of the set of the abelian varieties of that type and isogenous to a non trivial product of p.p.a.v.'s is $\leq 4$ if $g=3$, it is $\leq 7$ if $g=4$, it is $\leq 11$ if 
$g=5 \ldots ;$ thus if we consider the case $g=5$ and $d=3$ we find a counterexample to the conjecture.

Acknowledgements. I thank Prof. F. Catanese for some helpful discussions. This research was carried through in the realm of the AGE Project HCMERBCHRXCT940557 and of the 40\% MURST Program "Geometria algebrica".

\section{REFERENCES}

[B-L-R] Ch. Birkenhake, H. Lange and S. Ramanan, Primitive line bundles on Abelian threefolds, Manuscripta Math., 81 (1993), 299-310.

[F-R] H. Farkas and H. RaUCH, Theta Functions with Applications to Riemann Surfaces, The Williams \& Wilkıns Company, 1974.

[Ko] S. KorzumI, Theta relations and projectıve normality of abelian varietıes, Amer. J. Math., 98 (1976), 865-889.

[L-B] H. Lange and Ch. Birkenhake, Complex Abelian Varieties, Springer Verlag, 1992.

[Laz] R. LAZARSFELD, Projectivité normale des surfaces abéliannes, redigé par O. Debarre. prépublication No. 14 Europroj-C.I.M.P.A., Nice, 1990.

[Lef] S. LEFSCHETZ, On certain numerical invariants of algebraic varieties with applications to abelian varieties, Trans. Amer. Math. Soc., 22 (1921), 327-482.

[M] D. MumFord, Varietıes defined by quadratıc equations, Questionı sulle Varıetà Algebrıche, Corsı C.I.M.E., Edizionı Cremonese, Roma, 1970, 29-100.

[Oh1] A. OнвUсні, Some remarks on ample line bundles on abelian varieties, Manuscripta Math., 57 (1987), 225-238.

[Oh2] A. OHBUCHI, A note on the normal generation of ample line bundles on abelian var1eties, Proc. Japan Acad., 64 (1988), 119-120.

[R] E. RuBEI, Projective normality of abelian varieties with a line bundle of type $(2,$.$) , Boll.$ Unione Mat. Ital. Sez. B Artic Ric. Mat. (8), 1 (1998), 361-367.

VIA IV NOVEMBRe N. 5

Piombino (LI) C.A.P. 57025

ITALIA

e-mail: rube1@mail.dm.unıpı.it 